\title{
Toward CP-even Neutrino Beam 8
}

\author{
A. Fukumi ${ }^{\dagger}$, I. Nakano ${ }^{\dagger}$, H. Nanjo ${ }^{\ddagger}$, N. Sasao ${ }^{\ddagger}$, \\ S. Sato $^{\dagger}$, and M. Yoshimura ${ }^{\dagger}$ \\ ${ }^{\dagger}$ Center of Quantum Universe and Department of Physics, Okayama University, \\ Tsushima-naka 3-1-1, Okayama 700-8530, Japan \\ †epartment of Physics, Kyoto University, \\ Kitashirakawa, Sakyo, Kyoto, 606-8502 Japan
}

\begin{abstract}
The best method of measuring $\mathrm{CP}$ violating effect in neutrino oscillation experiments is to construct and use a neutrino beam made of an ideal mixture of $\bar{\nu}_{e}$ and $\nu_{e}$ of monochromatic lines. The conceptual design of such a beam is described, together with how to measure the CP-odd quantity. We propose to exploit an accelerated unstable hydrogen-like heavy ion in a storage ring, whose decay has both electron capture and bound beta decay with a comparable fraction.
\end{abstract}

It is of great current interest, both to microphysics and to cosmology, to clarify how the matterantimatter asymmetry is generated. One key idea is the leptogenesis [1] in which the lepton asymmetry of the universe is first generated by $\mathrm{CP}$ violating lepton number violating processes, and is later converted to the baryon asymmetry via high temperature B- and L-violating electroweak processes. To advance this idea further, it is important to deepen our understanding on the origin of $\mathrm{CP}$ violation in the leptonic sector.

Measurement of $\mathrm{CP}$ violation in the neutrino sector is thus one of the most fundamental problems facing physics beyond the standard particle physics. In this note we develop a concept of CP-even neutrino beam, which serves this purpose. Moreover, it is one of the most important experimental issues in neutrino physics to determine the relevant $\mathrm{CP}$ parameter in neutrino oscillation experiments.

The ideal neutrino beam for CP measurement would be a mixture of monochromatic $\nu_{e}$ and $\bar{\nu}_{e}$ beam for which the detector response is symmetrical, producing after the oscillation equal numbers of $\mu^{ \pm}$if CP is conserved. Such a beam is referred to as a CP-even neutrino beam in this paper. Our proposal is to simultaneously use the monochromatic neutrino $\nu_{e}$ of electron capture (EC) and anti-neutrino $\bar{\nu}_{e}$ of bound beta $(b \beta)$ decay from hydrogen-like heavy ions. Such ions do exist, for example [2]

$$
{ }_{49}^{114} \mathrm{In}^{48+},{ }_{47}^{110} \mathrm{Ag}^{46+},{ }_{47}^{108} \mathrm{Ag}^{46+},{ }_{45}^{104} \mathrm{Rh}^{44+} .
$$

The concept of CP-even neutrino beam is realized, to a large extent, by this kind of beam. It is an extension of the ideas of monochromatic neutrino beam using EC proposed in [3], 4, and beta beam proposed in [5], 6].

\footnotetext{
${ }^{\S}$ Work supported in part by the Grant-in-Aid for Science Research from the Ministry of Education, Science and Culture of Japan No.18654046, No.18684008, and No. 13135207
} 
The hydrogen-like heavy ion of this kind has $b \beta$ channels in addition to continuum beta $(c \beta)$ decay and EC, and thus produces monochromatic $\bar{\nu}_{e}$ and $\nu_{e}$ beams once accelerated in a storage ring. One can arrange all lines and a part of the continuum neutrino energy to fall into an optimal range for detection: above $110 \mathrm{MeV}$ of the muon production, but below multi-pion production in detector placed at a distance. We shall argue that this provides an excellent opportunity of precision experiments to determine the $\mathrm{CP}$ violation phase $\delta$ [7] as well as the mixing angle $\theta_{13}$.

Although not exhaustive by any means, a systematic method of search for candidate ions is briefly described, starting from data of neutral atoms. Since we momentarily ignore $c \beta$ contribution for detection, one important measure for the $\mathrm{CP}$-even beam is a large $\mathrm{EC}$ rate of neutral atom. This rate is almost the same for the case of the heavy ions. It is then important to look for calculated $b \beta$ rate of about twice of EC rate, since cross sections for $\nu$ and $\bar{\nu}$ has this ratio (for more details, see below).

A possibility of the bound beta decay that produces monochromatic neutrino has been considered theoretically [8], and their dramatic example that becomes possible only for highly ionized atoms has been demonstrated experimentally [9]. Relative strength of different neutrino lines is proportional to the atomic wave function squared at the nucleus. This factor for the s-wave state of the principal quantum number $n$ is $\left|\psi_{n s}(0)\right|^{2}=\left(Z / n a_{B}\right)^{3} / \pi$ (using for simplicity the solution of the Schrödinger equation) for hydrogen-like atoms of charge $Z e$. The ratio of the bound to the continuum contribution is given by

$$
r_{B}=\pi \sum_{n} N_{n}\left(\frac{3.7 k e V Z}{n Q_{c \beta}}\right)^{3}\left(\frac{Q_{c \beta}+\Delta_{\mathrm{BE}}}{Q_{c \beta}}\right)^{2} K^{-1}\left(\frac{m_{e}}{Q_{c \beta}}\right),
$$

with $N_{n}$ the multiplicity factor of available levels and $\Delta_{\mathrm{BE}}$ the difference of binding energy in neighboring ions. Here $K(x)$ is related to the phase space integral of the continuum contribution. When the effect of Coulomb distortion of wave functions (the Fermi integral) is ignored, this function is in the range, $K(x)=0.15 \sim 0.74$ for $x=1 / 2 \sim 2$. When variation of the level difference $Q_{c \beta}+\Delta_{\mathrm{BE}}$ with $n$ is small, the fraction of $1 s$ contribution is $1 /(2 \zeta(3)-1) \sim 0.71,2 s$ contribution 0.18 , and the rest 0.11 . To obtain a large $b \beta$ rate, $r_{B}=O[1]$ is required; for instance, with $Q_{c \beta}+\Delta_{\mathrm{BE}}=m_{e}$, the bound ratio $r_{B}=1$ corresponds to $Z=57$. We thus look for heavy atoms of large $Z$.

We found the best candidate ion to be ${ }_{47}^{108} \mathrm{Ag}^{46+}$, and the next best one is ${ }_{49}^{114} \mathrm{In}^{48+}$. The neutrino and anti-neutrino energy spectrum from decay of ${ }_{47}^{108} \mathrm{Ag}^{46+}$ at rest is shown in Figure 1 . Effect of distorted plane wave under the nuclear Coulomb potential is important and included in the form of the Fermi integral.

Table 1 gives their monochromatic energies and the endpoint energy of the continuum, along with their fractional contributions. To compare the line contribution with the differential continuum spectrum, we divided the line strength by an energy resolution factor $\Delta E$, which is taken $10 \mathrm{keV}$ in Figure 1. We denoted decay contributions to excited daughter nuclei by ${ }^{*}$ in Figure 1 and Table 1. It is important to use the wave function of the Dirac equation rather than the Schrödinger equation, since the wave function at nucleus is sensitive to the short distance behavior. Most conspicuous lines from ${ }^{108} \mathrm{Ag}^{46+}$ have fractions; 0.020 adding bound $\mathrm{K}$-, L- and M-beta decays all to the ground nuclear level, and 0.0096 for $E C+E C^{*}$.

All promising candidates we know of are listed in Table 2, along with their characteristic features.

$\mathrm{CP}$ violating effects are present only for the appearance experiment [10], hence we need to detect the muon neutrino from the oscillation, $\nu_{e} \rightarrow \nu_{\mu}$ and $\bar{\nu}_{e} \rightarrow \bar{\nu}_{\mu}$, thus to set the neutrino 


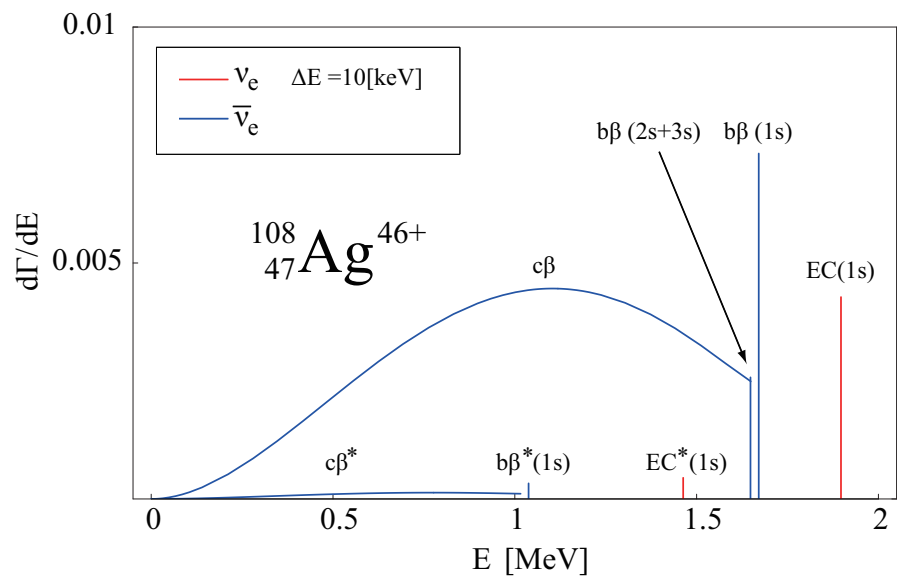

Figure 1: Beta and EC neutrino spectrum from ${ }^{108} \mathrm{Ag}^{46+}$

Table 1: Branching ratio of $c \beta, b \beta$, and EC from ${ }_{47}^{108} \mathrm{Ag}^{46+}$ decay

\begin{tabular}{ccc}
\hline \hline decay mode & neutrino energy $[\mathrm{MeV}]$ & branching ratio \\
\hline \hline$c \beta$ & 1.649 & 0.9479 \\
$c \beta^{*}$ & 1.016 & 0.0174 \\
\hline$b \beta(1 s)$ & 1.671 & 0.0149 \\
$b \beta(2 s)$ & 1.648 & 0.0041 \\
$b \beta(3 s)$ & 1.645 & 0.0012 \\
\hline $\mathrm{EC}(1 \mathrm{~s})$ & 1.897 & 0.0087 \\
\hline $\mathrm{EC}^{*}(1 s)$ & 1.463 & 0.0009 \\
\hline \hline
\end{tabular}

Table 2: Neutrino energy (E) and branching ratio (BR) of $c \beta, b \beta$ and EC

\begin{tabular}{|c|c|c|c|c|}
\hline ion & \multicolumn{2}{|c|}{${ }_{49}^{114} \operatorname{In}^{48+}$} & \multicolumn{2}{|c|}{${ }_{47}^{110} \mathrm{Ag}^{46+}$} \\
\hline half-life & \multicolumn{2}{|c|}{$70.9[\mathrm{sec}]$} & \multicolumn{2}{|c|}{$24.4[\mathrm{sec}]$} \\
\hline & $E[\mathrm{MeV}$ & BR & $E[\mathrm{MeV}]$ & $\mathrm{BR}$ \\
\hline$c \beta$ & 1.99 & 0.981 & 2.89 & $\overline{0.947}$ \\
\hline$b \beta(1 s)$ & 2.01 & 0.012 & 2.91 & 0.005 \\
\hline $\mathrm{EC}(1 \mathrm{~s})$ & 1.43 & 0.002 & 0.87 & 0.001 \\
\hline
\end{tabular}

\begin{tabular}{c|cc|cc}
\hline \hline ion & \multicolumn{2}{|c|}{${ }_{47}^{108} \mathrm{Ag}^{46+}$} & \multicolumn{2}{c}{${ }_{45}^{104} \mathrm{Rh}^{44+}$} \\
\hline \hline half-life & \multicolumn{2}{|c|}{$2.36[\mathrm{~min}]$} & \multicolumn{2}{c}{$42[\mathrm{sec}]$} \\
\hline & $E[\mathrm{MeV}]$ & $\mathrm{BR}$ & $E[\mathrm{MeV}]$ & $\mathrm{BR}$ \\
\hline$c \beta$ & 1.65 & 0.948 & 2.44 & 0.971 \\
$b \beta(1 s)$ & 1.67 & 0.015 & 2.46 & 0.006 \\
$\mathrm{EC}(1 \mathrm{~s})$ & 1.90 & 0.009 & 1.12 & 0.002 \\
\hline \hline
\end{tabular}


energy above muon production. The appearance probability for $\nu_{e} \rightarrow \nu_{\mu}\left(\right.$ and $\left.\bar{\nu}_{e} \rightarrow \bar{\nu}_{\mu}\right)$ is to a good approximation given by

$$
\begin{aligned}
& P_{\nu_{e} \nu_{\mu}\left(\bar{\nu}_{e} \bar{\nu}_{\mu}\right)}=s_{23}^{2} \sin ^{2} 2 \theta_{13} \sin ^{2} \frac{\delta m_{13}^{2} L}{4 E} \\
& +c_{23}^{2} \sin ^{2} 2 \theta_{12} \sin ^{2} \frac{\delta m_{12}^{2} L}{4 E} \\
& +J \cos \left( \pm \delta-\frac{\delta m_{13}^{2} L}{4 E}\right) \frac{\delta m_{12}^{2} L}{4 E} \sin \frac{\delta m_{13}^{2} L}{4 E},
\end{aligned}
$$

with $J=c_{13} \sin 2 \theta_{12} \sin 2 \theta_{23} \sin 2 \theta_{13}$. We note that for the range of $\left(\delta m_{12}^{2} /\left(4 m_{13}^{2}\right)\right)^{2}<\theta_{13}<$ $\delta m_{12}^{2} /\left(4 m_{13}^{2}\right)$, which implies roughly $\theta_{13}=0.0025 \sim 0.05$, the last CP sensitive term in eq.(3) is the largest. The matter effect that may mimic CP effects is negligible at this low energy. To determine $\theta_{13}$ and $\delta$ with precision, choice of the detector location $L$ is important. Taking account of the neutrino flux factor $\propto 1 / L^{2}$, three terms in the oscillation probability (3) have different $L$-dependence, $\propto L^{-2}, L^{0}, L^{-1}$ respectively, when the phase $\varphi=\delta m_{13}^{2} L /(4 E)$ is near the oscillation peak. To maximize simultaneously the flux at the detector and sensitivity to CP parameter $\delta$, the best location is at the first peak $\varphi=\pi / 2$. With this choice, the last term in (3) becomes proportional to $\pm J \sin \delta$. Thus the symmetric combination of measured quantities $P_{\nu_{e} \nu_{\mu}}+P_{\bar{\nu}_{e} \bar{\nu}_{\mu}}$ is sensitive to $\theta_{13}$, while the asymmetric combination $P_{\nu_{e} \nu_{\mu}}-P_{\bar{\nu}_{e} \bar{\nu}_{\mu}}$ to the CP violation parameter $J \sin \delta$. This choice fixes the relation between $E$ and $L$ to be $L / E \simeq 517 \mathrm{~km} / 1000 \mathrm{MeV}$. In Figure 2 we plot the oscillation probability $P\left(\nu_{e} \rightarrow \nu_{\mu}\right)$ as a function of $\delta$ for three values of $\sin ^{2} 2 \theta_{13}=0.1,0.05,0.01$.

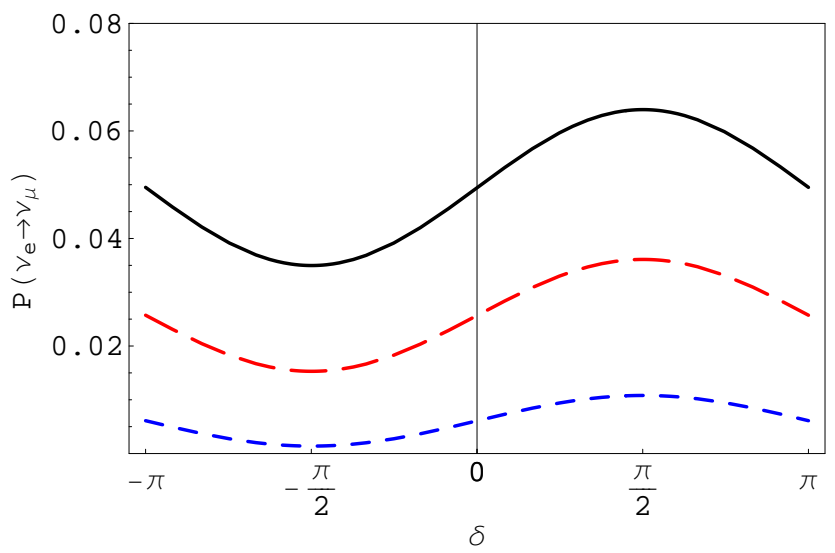

Figure 2: Oscillation probability $P_{\nu_{e} \nu_{\mu}}$ at the first peak $\varphi=\pi / 2$ for $\sin ^{2} 2 \theta_{13}=0.1,0.05,0.01$ (from top to bottom). $P_{\bar{\nu}_{e} \bar{\nu}_{\mu}}$ is obtained with $\delta \rightarrow-\delta$.

The neutrino energy $E$ can be chosen at will by adjusting the acceleration $\gamma$. There are many practical factors to be considered; here we select them mainly from the viewpoint of neutrino detection. The cross section becomes larger as the energy goes up, but distinction between $\mu^{+}$and $\mu^{-}$, mandatory to establish genuine $\mathrm{CP}$ violating effects, becomes harder. Below the single-pion production threshold, backgrounds due to $\pi^{ \pm}$'s are absent; but the cross section is small and the inevitable Fermi motion smears out kinematic relation between $\mu^{ \pm}$energy and scattering angle. Our optimal choice is around $1000 \mathrm{MeV}$, which is somewhat above the multi-pion production threshold. 
At this energy, the cross section for quasi-elastic process $\left(\nu_{\mu} n \rightarrow \mu^{-} p\right.$ or $\left.\bar{\nu}_{\mu} p \rightarrow \mu^{+} n\right)$ is $1.5-3$ times as large as for the single-pion production cross section. Other channels such as multi-pion productions are negligible.

We note, however, that optimal choice of the neutrino energy may be changed in actual experiments, depending upon chosen technologies of accelerators as well as detectors.

The concept of CP-even beam is both detector and neutrino energy dependent, since the $\nu_{\mu}$ and $\bar{\nu}_{\mu}$ cross sections are different for different targets, and the line strengths are different at different energies. One may define the CP-evenness $\eta$, using a beam flux $\mathcal{F}\left(\nu_{e}\right)$ from ions weighted by the cross section $\sigma\left(\nu_{\mu}\right)$ at definite neutrino energies;

$$
\eta(E ; \gamma)=\frac{\mathcal{F}\left(\nu_{e}\right) \sigma\left(\nu_{\mu}\right)-\mathcal{F}\left(\bar{\nu}_{e}\right) \sigma\left(\bar{\nu}_{\mu}\right)}{\mathcal{F}\left(\nu_{e}\right) \sigma\left(\nu_{\mu}\right)+\mathcal{F}\left(\bar{\nu}_{e}\right) \sigma\left(\bar{\nu}_{\mu}\right)}
$$

In order to determine $\eta$ for actual beams, we need to know two quantities: one is the relative intensity of $\nu_{e}$ and $\bar{\nu}_{e}$ flux, and the other is the relative $\nu_{\mu}$ and $\bar{\nu}_{\mu}$ cross section. The former quantity, $\mathcal{F}\left(\nu_{e}\right) / \mathcal{F}\left(\bar{\nu}_{e}\right)$, may be estimated from the measured branching ratio of EC and $\beta$-decay. As to the latter quantity, $\sigma\left(\nu_{\mu}\right) / \sigma\left(\bar{\nu}_{\mu}\right)$, we must include a detector response function (efficiency etc.) in reality. In this case, the ratio would be best determined in a separate experiment with an identical detector component. As an example, let us take ${ }^{108} \mathrm{Ag}^{46+}$ as a beam, and an iron detector. The uncertainty of $\mathcal{F}\left(\nu_{e}\right) / \mathcal{F}\left(\bar{\nu}_{e}\right)$ is $\sim 7 \%$ at present [2] and CP-evenness $\eta \approx 0.09$ selecting the major four lines, neglecting energy dependence of cross sections, and including the effect of Fermi motion. If the contribution of $c \beta$ integrated over the energy interval $\approx 8 \mathrm{keV}$ below the threshold is added, the CP-evenness $\eta \approx 0$. We have so far discussed the idea of a single-ion beam. From the point of larger rates, a multi-ion beam is equally interesting. The multi-ion beam consists of a simultaneous or time sharing circulation of two different ions of nearly equal $Z / A$ 's, each suitable for the bound beta and EC, individually having higher rates. For this beam it is important to identify and use ions of largest rates for $b \beta$ and EC, respectively. Both candidates of larger $b \beta$ or EC rates than the single-ion isotopes are numerous. We only mention two of them; ${ }_{48}^{122} \mathrm{Cd}^{48+}$ for $b \beta$, and ${ }_{70}^{152} \mathrm{Yb}$ for EC. The event rate of neutrino detection depends on the isotope factor of $\Gamma / Q$ with $\Gamma$ the partial $b \beta$ or EC decay rate. The isotope ${ }_{48}^{122} \mathrm{Cd}^{48+}$, for instance, has the 1 s $b \beta$ event rate larger by a factor $\approx 12$ than the $b \beta$ rate of ${ }_{47}^{108} \mathrm{Ag}^{46+}$, and ${ }_{70}^{152} \mathrm{Yb}$ EC rate is larger by $\approx 600$ than the corresponding EC rate of ${ }_{47}^{108} \mathrm{Ag}^{46+}$. The mixed beam made of ${ }_{48}^{122} \mathrm{Cd}^{48+}$ (for $\bar{\nu}_{e}$ ) and ${ }_{70}^{152} \mathrm{Yb}^{60+}$ (for $\nu_{e}$ ) has similar $Z / A$, and it may be possible to accelerate both of them simultaneously. Their monochromatic neutrino energies are $3.0 \mathrm{MeV}(1 s \mathrm{~b} \beta)$ for ${ }_{48}^{122} \mathrm{Cd}^{48+}$ and $5.0 \mathrm{MeV}$ ( $1 s \mathrm{EC}$ ) for ${ }_{70}^{152} \mathrm{Yb}$, hence the time sharing circulation might be more appropriate. This option of multi-ion beam opens more varieties toward the $\mathrm{CP}$-even beam and should be kept in mind for further study.

After submitting this paper, we have noticed that the measured EC decay rate of hydrogen-like ${ }^{140} \mathrm{Pr}^{58+}$ ion is about $50 \%$ larger than that of helium-like ${ }^{140} \mathrm{Pr}^{57+}$ ion [11, due to angular momentum restriction of both leptons and nuclei. [12] [13] A similar mechanism may occur in our system ${ }^{108} \mathrm{Ag}^{46+},{ }^{110} \mathrm{Ag}^{46+},{ }^{114} \mathrm{In}^{48+}$ thereby enhancing our EC rates of Tables 1 and 2.

In summary, we proposed a new concept to implement the experimental method optimal for determination of the CP parameter of neutrino mixing by using monochromatic neutrino beams both from electron capture and bound beta decay. Feasibility of such beams hinges crucially on production and storage of high intensity unstable nuclear beams, and innovative works towards this direction 
are needed.

\section{References}

[1] M. Fukugita and T. Yanagida: Phys. Lett. 174B (1986) 45. W. Buchmuller and M. Plumacher: Int. J. Mod. Phys. A15 (2000) 5047. G. F. Giudice, A. Notari, M. Raidal, A. Riotto, and A. Strumia: Nucl. Phys. B685 (2004) 89.

[2] Evaluated Nuclear Structure Data File (ENSDF), maintained by the National Nuclear Data Center, Brookhaven National Laboratory (2006).

[3] J. Sato: Phys. Rev. Lett. 95 (2005) 131804 and hep-ph/0503144.

[4] J. Bernabeu and C. Espinoza: hep-ph/0605132; J. Bernabeu, J. Burguet-Castell, C. Espinoza and M. Lindroos: JHEP 0512 (2005) 014 and hep-ph/0505054.

[5] P. Zucchelli: Phys. Lett. B532 (2002) 166, and hep-ex/0107006.

[6] For a status of beta beam, B. Autin, M. Benedikt, M. Grieser, S. Hancock, H. Haseroth, A. Jansson, U. Koster, M. Lindroos, S. Russenschuck, and F. Wenander: J. Phys. G29 (2003) 1785, and http://cern.ch/beta-beam.

[7] We follow the notation of A. Cervera, A. Donini, M. B. Gavela, J. J. Gomez Cadenas, P. Hernandez, O. Mena, and S. Rigolin: Nucl. Phys. B579 (2000) 17.

[8] J.N. Bahcall: Phys. Rev. 124 (1961) 495, and references therein.

[9] M. Jung, F. Bosch, K. Beckert, H. Eickhoff, H. Folger, B. Franzke, A. Gruber, P. Kienle, O. Klepper, W. Koenig, C. Kozhuharov, R. Mann, R. Moshammer, F. Nolden, U. Schaaf, G. Soff, P. Spadtke, M. Steck, Th. Stohlker, and K. Summerer: Phys. Rev. Lett. 69 (1992) 2164.

[10] For instance, T. Yanagida and M. Yoshimura: Progr. Theor. Phys. 64 (1980) 1870.

[11] Yu. A. Litvinov, F. Bosch, H. Geissel, J. Kurcewicz, Z. Patyk, N. Winckler, L. Batist, K. Beckert, B. Boutin, C. Brandau, L. Chen, C. Dimopoulou, B. Fabian, T. Faestermann, A. Fragner, L. Grigorenko, E. Haettner, S. Hess, P. Kienle, R. Knoebel, C. Kozhuharov, S. A. Litvinov, L. Maier, M. Mazzocco, F. Montes, G. Munzenberg, A. Musumarra, C. Nociforo, F. Nolden, M. Pfutzner, W. R. Plass, A. Prochazka, R. Reda, R. Reuschl, C. Scheidenberger, M. Steck, T. Stoehlker, S. Torilov, M. Trassinelli, B. Sun, H. Weick, and M. Winkler: Phys. Rev. Lett. 99 (2007) 262501.

[12] L. M. Folan and V. I. Tsifrinovich: Phys. Rev. Lett. 74 (1995) 499.

[13] Z. Patyk, J. Kurcewicz, F. Bosch, H. Geissel, Y. A. Litvinov and M. Pfutzner: Phys. Rev. C77 (2008) 014306. 